\title{
Thermal transport properties of disordered spin-1/2 systems
}

\author{
Fabian Heidrich-Meisner ${ }^{\text {a }}$ \\ ${ }^{a}$ Technische Universität Braunschweig, Institut für Theoretische Physik, Mendelssohnstrasse 3, D-38106 Braunschweig, \\ Germany
}

\begin{abstract}
This work studies heat transport of bond-disordered spin- $1 / 2$ chains. As an example, the $X X$ case is analyzed, which corresponds to a model of noninteracting spinless fermions. Within the fermion representation, the singleparticle eigenenergies are determined numerically, which allow one to compute transport coefficients. Since the ballistic transport properties of a clean chain are destroyed by disorder, the focus is on the frequency dependence of the thermal conductivity and on a qualitative comparison with the spin conductivity, both at finite temperatures.
\end{abstract}

Key words: Quantum spin systems, Transport properties, Disorder PACS: $75.10 . \mathrm{Jm}, 74.25 . \mathrm{Fy}, 75.40 . \mathrm{Mg}$

Motivated by recent experiments on transport properties of transition metal oxides [1], intense theoretical work has recently been devoted to the study of heat conduction in one-dimensional spin- $1 / 2$ systems such as the Heisenberg chain, frustrated chains, and spin ladders [2]. Among these systems, the anisotropic spin- $1 / 2$ chain exhibits ballistic thermal transport properties at all temperatures $T$ due to its integrability [3]. The thermal conductivity diverges in the homogeneous case, signaled by a finite thermal Drude weight [4]. Here, the usual decomposition of the real part of the thermal conductivity $\kappa$ into the Drude weight $D_{\text {th }}$ and the regular part is utilized: $\operatorname{Re} \kappa(\omega)=$ $D_{\text {th }} \delta(\omega)+\kappa_{\text {reg }}(\omega), \omega$ being the frequency. Ballistic transport is expected to be destroyed by randomness in the exchange couplings, resulting in a vanishing Drude weight. Therefore, one is interested in the regular part $\kappa_{\text {reg }}(\omega)$, from which the dc-conductivity can be extracted by extrapolating to $\omega=0$.

In this contribution, the effect of bond disorder on the thermal conductivity of $X X$ chains is stud-

\footnotetext{
* Corresponding author. Tel: +49-531-3915184; Fax: +49-5313915833

Email address: f.heidrich-meisner@tu-bs.de (Fabian Heidrich-Meisner).
}

ied numerically. This limiting case of the anisotropic spin- $1 / 2$ chain corresponds to free spinless fermions by means of a Jordan-Wigner transformation [5]. While particle transport in disordered fermion systems is a long-studied problem, closely related to the subject of localization (see, e.g., Ref. [6]), the thermal conductivity $\kappa$ in these models has attracted less attention. Using bosonization, predictions were made for the concentration dependence of the thermal conductivity of spin-1/2 chains [7], and it could be very interesting to compare this to numerical results. Here, results for the frequency dependence of $\kappa$ are presented for the case of off-diagonal disorder at finite temperatures.

The Hamiltonian in terms of spin- $1 / 2$ operators $S_{l}^{ \pm, z}$ acting on site $l$ and with periodic boundary conditions $(\mathrm{PBC})$ reads:

$$
H=\frac{1}{2} \sum_{l=1}^{N} J_{l}\left(S_{l}^{+} S_{l+1}^{-}+\text {h.c. }\right),
$$

where $N$ is the number of sites. It can equivalently be written in terms of fermionic operators $c_{l}^{\dagger}$ :

$$
H=\frac{1}{2} \sum_{l=1}^{N} J_{l}\left(c_{l}^{\dagger} c_{l+1}+\text { h.c. }\right) .
$$


In principle, a subtlety arises since the PBCs for the spin operators give rise to a nontrivial boundary term for the fermions, which depends on the number $\mathcal{N}$ of fermions [8]. This dependence, however, is neglected here, and PBC are imposed for all $\mathcal{N}$, since boundary effects can be expected to vanish for large system sizes.

Introducing a spinor $\psi^{\dagger}=\left(c_{1}^{\dagger}, \ldots, c_{N}^{\dagger}\right)$, the Hamiltonian can be written as $H=\psi^{\dagger} \mathcal{A} \psi$, where $\mathcal{A}$ is a symmetric $N \times N$ band matrix, with nonzero elements $A_{l, l+1}=J_{l} / 2$ and $A_{1, N}=J_{1} / 2$. Randomness in the $J_{l}$ is therefore called off-diagonal disorder, while a spatially varying magnetic field realizes diagonal disorder.

While a transformation to momentum space diagonalizes $H$ for the translationally invariant case $\left(J_{l}=J\right)$, the computation of single-particle eigenenergies is still straightforward for random couplings (see, e.g., [8]). By means of a unitary transformation $\mathcal{U}$ with $c_{l}=\sum_{\mu} \mathcal{U}_{l \mu} \eta_{\mu}$ that diagonalizes the matrix $\mathcal{A}$, the Hamiltonian can be written as $H=\sum_{\mu} \epsilon_{\mu} \eta_{\mu}^{\dagger} \eta_{\mu}$, where $\epsilon_{\mu}$ are the single-particle eigenenergies.

The energy current operator corresponding to Eq. (2) is $\mathcal{J}=(i / 4) \sum_{l}\left(c_{l}^{\dagger} c_{l+2}-\right.$ h.c. $)=i \sum_{\mu \nu} \eta_{\nu}^{\dagger} \mathcal{J}_{\nu \mu} \eta_{\mu}$ Within linear response theory, $\kappa_{\mathrm{reg}}(\omega)$ is given by [6]:

$\kappa_{\mathrm{reg}}(\omega)=\frac{\beta}{\omega} \sum_{\epsilon_{\mu} \neq \epsilon_{\nu}}\left|\mathcal{J}_{\nu \mu}\right|^{2}\left[f\left(\epsilon_{\mu}\right)-f\left(\epsilon_{\nu}\right)\right] \delta(\omega-\Delta \epsilon)$,

where $f(\epsilon)=1 /(\exp (\beta \epsilon)+1)$ denotes the Fermifunction, $\beta=1 / T$, and $\Delta \epsilon=\epsilon_{\nu}-\epsilon_{\mu}$.

As an example, a Gaussian distribution of random couplings is considered: $P\left(J_{l}\right) \propto e^{-\left(J_{l}-J\right)^{2} / w^{2}}$, with $J=1$ and $w=0.2$. The choice of the distribution does not affect the results, quantitatively consistent results for $\kappa(\omega)$ are obtained with other $P_{l}$ (e.g. box or binary distribution) by fixing the first moments of the distribution function. Figure 1 shows results for $\kappa_{\text {reg }}(\omega)$ [panel(b)] and, for comparison, $\sigma_{\text {reg }}(\omega)$ [panel(a)], which is the spin conductivity corresponding to the current operator $\mathcal{J}_{\mathrm{s}}=(i / 2) \sum_{l}\left(c_{l}^{\dagger} c_{l+1}-\right.$ h.c. $)$. The parameters are $T / J=0.5$ and $N=1000,5000$. An imaginary broadening of $10^{-4}$ was used. The overall form of both quantities is quite similar and resembles that known for $\sigma_{\mathrm{reg}}(\omega)$ [9]. Moreover, both curves exhibit a maximum at roughly the same frequency $(\omega / J \approx 0.015$ for the parameters of Fig. 1). Both finite-size effects and statistical fluctuations are small in the low-frequency limit.

The behavior of $\kappa_{\text {reg }}(\omega)$ at low frequencies is of particular interest as it determines the dc-conductivity (see Refs. [10] for the case of clean spin systems). While the Drude weights vanish in the presence of disorder, the results of this work indicate finite dc-conductivities for both spin and thermal transport at finite temperatures. This is illustrated in the inset of Fig. 1. A more detailed analysis of the finite-size scaling as well as the temperature dependence will be presented elsewhere.

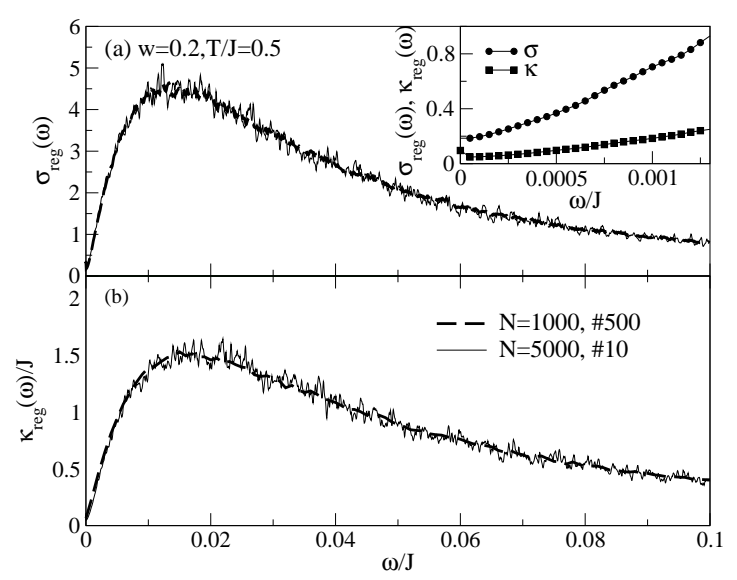

Fig. 1. Spin [panel (a)] and thermal conductivity [panel (b)] of an $X X$ chain with a Gaussian distribution of random couplings $J_{l}$, centered around $J=1$ and with a width of $w=0.2$ (see text). Curves for $N=1000(5000)$ sites and 500(10) random realizations for the same parameters are displayed. An imaginary broadening of $10^{-4}$ is used. The inset shows a blow-up of the low frequency region for the larger system size.

The author acknowledges fruitful discussions with W. Brenig, B. Büchner, C. Hess, and A. Honecker. This work was supported by the Deutsche Forschungsgemeinschaft through SPP 1073 and grant HE-5242/1-1.

References

[1] See, e.g.: A. V. Sologubenko et al., Phys. Rev. Lett. 84 (2000) 2714; C. Hess et al., Phys. Rev. B 64 (2001) 184305; K. Kudo et al., J. Phys. Soc. Jpn. 70 (2001) 437.

[2] See: X. Zotos and P. Prelovšek: in Strong Interactions in Low Dimensions, Kluwer Academic Publishers, 2004; and further references therein.

[3] X. Zotos, F. Naef, and P. Prelovšek, Phys. Rev. B 55 (1997) 11029.

[4] A. Klümper and K. Sakai, J. Phys. A 35 (2002) 2173; K. Sakai and A. Klümper J. Phys. A 36 (2003) 11617; F. Heidrich-Meisner et al., Phys. Rev. B 66 (2002) 140406; ibid. 68 (2003) 134436; ibid. 71 (2005) 184415.

[5] G. D. Mahan, Many-Particle Physics, Plenum Press, New York London, 1990.

[6] B. Kramer and A. MacKinnon, Rep. Prog. Phys. 56 (1993) 1469 .

[7] C. I. Kane and M. P. A. Fisher, Phys. Rev. Lett. 76 (1996) 3192; A. V. Rozhkov and A. L. Chernyshev, Phys. Rev. Lett. 94 (2005) 087201.

[8] N. Laflorencie and H. Rieger, Eur. Phys. J. B 40 (2004) 201.

[9] O. Motrunich, K. Damle, and D. A. Huse, Phys. Rev. B 63 (2001) 134424.

[10] X. Zotos, Phys. Rev. Lett. 92 (2004) 067202; F. HeidrichMeisner et al., Physica B 359-361 (2005) 1394. 